Notfall Rettungsmed 2017 $20: 97-99$

DOI 10.1007/s10049-017-0278-6

Online publiziert: 15 . Februar 2017

(c) Springer Medizin Verlag GmbH 2017

CrossMark

\author{
M. Bernhard' $\cdot$ C. Waydhas ${ }^{2,3} \cdot$ B. Hossfeld ${ }^{4}$ \\ 'Zentrale Notaufnahme, Universitätsklinikum Leipzig, Leipzig, Deutschland \\ ${ }^{2}$ Bergmannsheil, Berufsgenossenschaftliches Universitätsklinikum, Ruhr-Universität Bochum, Bochum, \\ Deutschland \\ ${ }^{3}$ Medizinische Fakultät, Universität Essen-Duisburg, Essen, Deutschland \\ ${ }^{4}$ Klinik für Anästhesiologie und Intensivmedizin, Sektion Notfallmedizin, Bundeswehrkrankenhaus Ulm, \\ Ulm, Deutschland
}

\title{
Vom Lebenszyklus der Medizinprodukte und Innovationen
}

„Medizinprodukte sind Instrumente, Apparate, Vorrichtungen [...] einschließlich [...] Software, die vom Hersteller zur Anwendung für Menschen mittels ihrer Funktionen zum Zwecke

a) der Erkennung, Verhütung, Überwachung, Behandlung oder Linderung von Krankheiten (z. B. Fieberthermometer, Katheter, Laborgeräte, Endoskope),

b) der Erkennung, Überwachung, Behandlung, Linderung oder Kompensierung von Verletzungen oder Behinderungen (z. B. Verbandsmaterial, orthopädische Hilfsmittel, Krankenpflegemittel, OP-Material),

c) der Untersuchung, der Ersetzung oder der Veränderung des anatomischen Aufbaus oder eines physiologischen Vorgangs (z. B. Prothesen, Implantate) oder

d) der Empfängnisregelung (z. B. Pressare, Kondome)

$\mathrm{zu}$ dienen bestimmt sind und deren bestimmungsgemäße Hauptwirkung im oder am menschlichen Körper weder durch pharmakologisch oder immunologisch wirkende Mittel noch durch Metabolismus erreicht wird, deren Wirkungsweise aber durch solche Mittel unterstützt werden kann."

Mit diesen Worten definiert $\$ 3$ Nr. 1 Medizinproduktegesetz (MPG) Medizinprodukte. Fernab von dieser komplexen Definition der Medizinprodukte setzt jeder in der Notfallmedizin
Tätige irgendwie und irgendwann $\mathrm{Me}$ dizinprodukte ein und ist damit auf Medizintechnik angewiesen.

Gemäß des Produkt-Prozess-Lebenszyklus-Modells nach James M. Utterback und William J. Abernathy [5] erleben neue Medizinprodukte zunächst eine Experimentierphase (fließende Phase), dann eine Übergangsphase und letztendlich eine spezifische Phase (• Abb. 1).

Bei einer Produktinnovation wird ein bisher nicht bestehendes Produkt neu entwickelt und weist daher eine hohe Innovationsrate auf. In der Übergangsphase kommt es zu einer stetigen Standardisierung und Effizienzsteigerung, dabei setzt sich das „dominante Design“ eines Produkts immer mehr auf dem Markt durch. Konkurrierende Unternehmen übernehmen dann dieses „dominante Design“ oder werden vom Markt verdrängt. In der spezifischen Phase ist das „dominante Design“ des Produkts dann fest auf dem Markt etabliert. Ein einmal entwickeltes Produkt muss aber über die Zeit immer wieder den Anforderungen des Markts angepasst werden, um wirtschaftlich erfolgreich zu sein. Nachdem sich das Produkt nun etabliert hat, folgt eine Phase der Produktvariation, entweder durch Prozessverbesserung oder durch die Entwicklung eines neuen Produkts (Produktverbesserung). Ein Beispiel hierfür ist die Entwicklung 1981 und Einführung 1983 der Larynxmaske von Sir Archie I. J. Brain [2], die seit 1988 in England und seit 1991 in Deutschland kommerziell im Handel erhältlich ist. Es folgt eine stetige Verbesserung und Variation des Produkts, um den Marktansprüchen zu genügen [6]: 1997 Intubationslarynxmaske [1] und LMA Unique, 2000 ProSeal Larynxmaske 2000 [3], 2007 LMA-Supreme [7].

Aber wie etabliert sich nun ein neu entwickeltes Medizinprodukt auf den Markt? Von Geoffrey A. Moore [4] wurde 1991 der sog. Technology Adoption Lifecycle (TALC) beschrieben (• Abb. 2). TALC bedeutet, dass eine dem Markt noch unbekannte Innovation durch Überzeugungsarbeit und viel Einsatz von Seiten des Herstellers der zukünftigen Zielgruppe an Kunden angeboten werden muss. TALC bezeichnet ein soziologisches Modell zur Beschreibung der Adaptation und der Akzeptanz eines neuen innovativen Produkts in verschieden motivierte Anwendergruppen. Dabei werden 5 Zielgruppen unterschieden.

Als erste Zielgruppe kommen die sog. Innovators (Technikfans) zum Zug. Diese Anwendergruppe aus beispielsweise Universitäts- und Schwerpunktkliniken zeichnet sich durch eine eigene intrinsische intensive Suche nach neuen Techniken aus, und dies bereits lange bevor die Kunden der anderen Zielgruppen auf die neuen Produkte aufmerksam werden. Dies führt dazu, dass die Produkte bereits den Innovators bekannt sind, bevor dieses Produkt durch ein strategisch gut platziertes Marketingprogramm überhaupt 


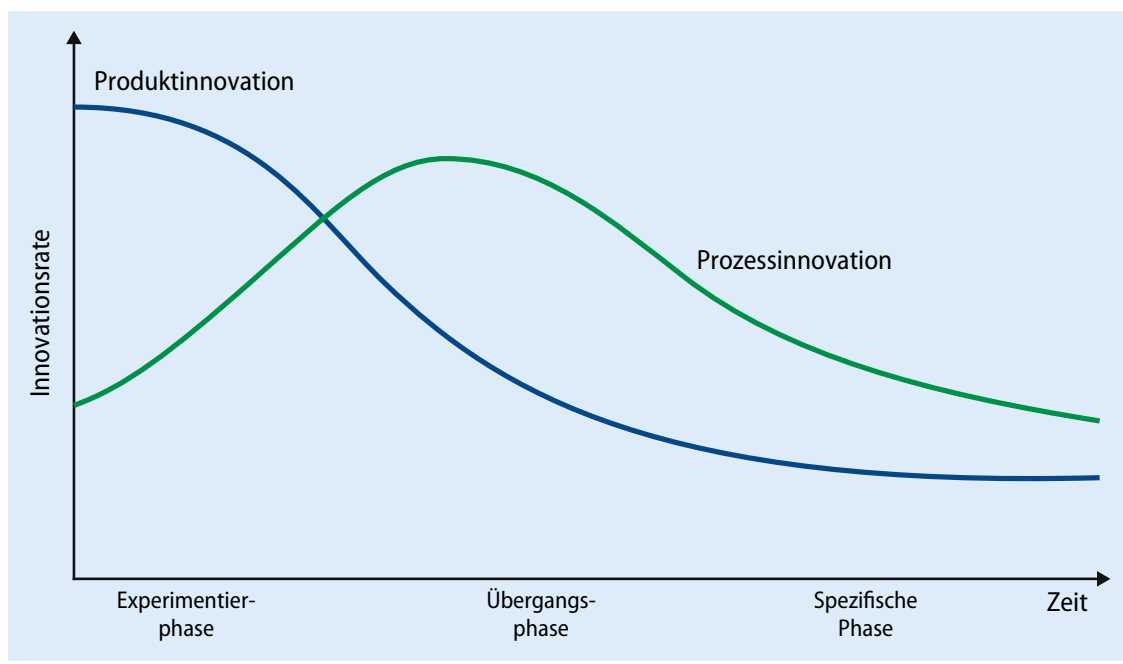

Abb. 1 A Produkt-Prozess-Lebenszyklus-Modell nach Utterback und Abernathy. (Mod. nach [5])

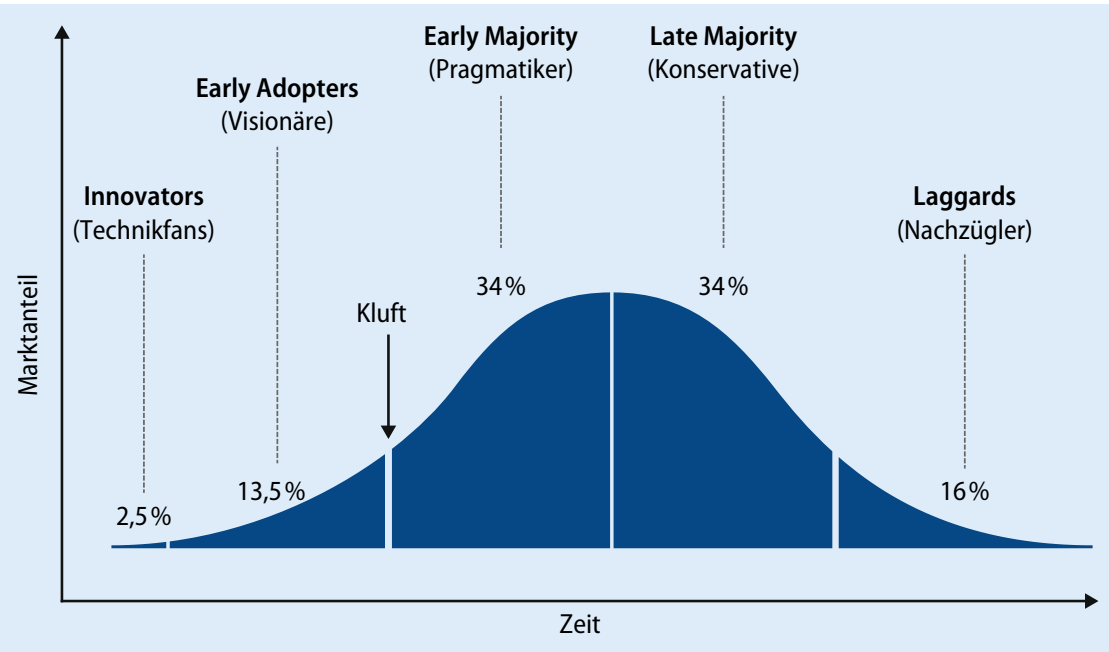

Abb. 2 ム Technology Adoption Lifecycle: Entwicklung und Einführung eines neuen Medizinprodukts. (Mod. nach [4])

beworben wurde. Die Medizinprodukteindustrie hat demnach mit dieser ersten Gruppe keinen besonders großen Aufwand. Aber Anteilig sind die Innovators nur eine sehr kleine Gruppe. Von dieser Gruppe geht aber eine relevante Informationsweitergabe an die weiteren Gruppen aus, da auf Kongressen und in Fachzeitschriften über diese neue Technik/das neue Produkt berichtet wird.

Die zweite Gruppe wird als Early Adopters (Visionäre) bezeichnet. Diese Anwendergruppe aus Fachkliniken möchte sich durch den Einsatz neuer Techniken einen medizinischen Vorteil für die Patientenbehandlung verschaffen und dies trotz anfänglicher „Kinderkrankheiten“ neuer Medizinprodukte. bern zu sichern. Dabei spielen für diese Anwendergruppe aber eher Absatzdaten und Wachstumsprognosen eine Rolle als für beide zuvor genannte Gruppen. Entscheidungen der Early Majority fußen auf den Erfahrungen der Innovators, mit klar nachgewiesenem Nutzen des avisierten Medizinprodukts. Die Kluft zwischen Innovators, Early Adopters und Early Majority gilt es für einen Medizinproduktehersteller $\mathrm{zu}$ überwinden, dies ist ein ganz entscheidendes Ziel, und hierfür ist nun ein deutlich höherer Marketingaufwand notwendig. Gelingt es nicht, die Gruppe der Pragmatiker von einem Medizinprodukt zu überzeugen, werden die anderen verbleibenden Anwendergruppen sich auch nicht für das Produkt interessieren und ein relevanter Marktanteil geht verloren bzw. wird nicht erschlossen.

Die Anwender aus der sog. Late Majority (Konservativen) stellen eine vergleichbar große Gruppe wie die Early Majority dar und machen zusammen mit diesen $80 \%$ des gesamten Markts (potenzielle Kunden) aus. Für diese Gruppe (z. B. Hausärzte, kleinere Krankenhäuser) muss das Risiko von Investitionen minimal sein, eine Technik etabliert und der Nutzen des Medizinprodukts klar belegt sein. Die letzte Gruppe der sog. Laggards (Nachzügler) zeigen erst dann Interesse an einem für sie neuen Produkt, wenn die bisher verwendete Technik nur noch schwer erhältlich ist oder die Anschaffung deutlich Zusatzkosten verursacht. Relevant für Marketingstrategien eines Unternehmens ist diese letzte Gruppe kaum.

Vor diesem Hintergrund muss sich jeder notfallmedizinisch tätige Anwender immer wieder mit dem Thema Medizinprodukte und dem aktuellen Stand der Technik auseinandersetzen. Die vorliegenden Leitthemen sollen es ermöglichen, die Entwicklung aktueller Medizinprodukte im Auge zu behalten. Gemäß dem etablierten $\mathrm{ABCDE}-\mathrm{Schema}$ werden dann folgende Themen behandelt:

- unter A (Atemweg) relevante Medizinprodukte zum Atemwegsmanagement durch Küßner et al.,

- unter B aktuelle Aspekte zu Notfallrespiratoren von Kumle et al., 
- unter C aktuell verfügbare Medizinprodukte zur intraossären Punktion von Weissleder et al. und das Notfallequipment zur Blutungskontrolle von Wölfl et al.,

- unter D Point-of-Care-Technologie von Betz et al., und

- unter E Techniken zum Wärmeerhalt durch Trentzsch et al.

Aufgrund der schnellen Lebenszyklen bereits bestehender und ganz neuer Produkte und dem hohen Innovationstempo auf dem Markt ist es kaum möglich, die Medizinprodukte eines Segments komplett darzustellen. Kontinuierlich kommen neue Produkte hinzu und machen eine ständige Neubewertung erforderlich. Wir hoffen, den Lesern mit diesem Leitthemenheft neue und aktuelle Medizinprodukte ebenso wie Innovationen vorstellen zu können.

Mit den besten Wünschen zu einem hohen Wirkungsgrad,

Ihre

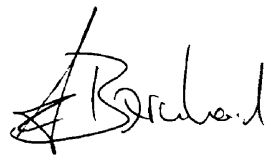

Michael Bernhard

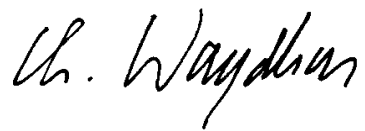

Christian Waydhas

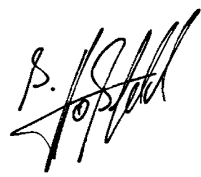

Björn Hossfeld

\section{Korrespondenzadresse}

PD Dr. med. habil. M. Bernhard

Zentrale Notaufnahme, Universitätsklinikum Leipzig

Liebigstraße 20, 04103 Leipzig, Deutschland Michael.Bernhard@medizin.uni-leipzig.de

Interessenkonflikt. M. Bernhard, C. Waydhas und B. Hossfeld geben an, dass kein Interessenkonflikt besteht.

\section{Literatur}

1. Bein B, Francksen $H$, Steinfarth $M$ (2011) Atemwegsmanagement - Supraglottische Atemwegshilfen. Anästhesiol Intensivmed Notfallmed Schmerzther 46:598-606

2. Brain AlK (1983) The laryngeal mask - A new concept in airway management. Br J Anaesth 55:801-805

3. Cook TM, Lee G, Nolan JP (2005) The ProSealTM laryngeal mask airway: a review of the literature. Can J Anaesth 52:739-760

4. Moore GA (1991) Crossing the chasm: marketing and selling high-tech products to mainstream customers (collins Buisness essentials). HarperBusiness, New York

5. Utterback JM, Abernathy WJ (1975) A dynamic model of process and product innovation. Omega (Westport) 3:639-656

6. Van Zundert TCRV, Brimacombe JR, Ferson DZ, Bacon DR, Wilkinson DJ (2012) Archie Brain: celebrating 30 years of development in laryngeal mask airways. Anaesthesia 67:1375-1385

7. Wong DT, Yang JJ, Jagannathan N (2012) Brief review: The LMA Supreme ${ }^{\mathrm{TM}}$ supraglottic airway. Can J Anesth 59:483-493

\section{Traumatische Verletzungen der Halswirbelsäule}

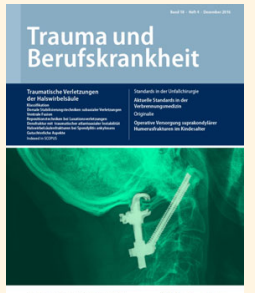

Die traumatischen Verletzungen der Halswirbelsäule umfassen neben den Verletzungen in Folge eines Hochrasanz-Traumas auch die soge-

nannten Niedrigenergie-Traumen, meist verursacht durch „einfache Stürze" älter werdender Patienten.

Das Leitthemenheft 4/2016 der Trauma und Berufskrankheit soll Ihnen einen Überblick der aktuellen operativen Therapieoptionen der häufigsten zervikalen Verletzungen geben.

Lesen Sie im Schwerpunktheft mehr zu folgenden Themen:

- Klassifikation und Gutachterliche Aspekte

- Dorsale Stabilisierungstechniken bei subaxialen Verletzungen

- Ventrale Fusion

- Repositionstechniken bei Luxationsverletzungen

- Densfraktur mit begleitender traumatischer atlantoaxialer Instabilität

- Frakturen der Halswirbelsäule bei Spondylitis ankylosans

Bestellen Sie diese Ausgabe zum Preis von 50,- EUR zzgl. Versandkosten bei Springer Customer Service Center, Kundenservice Zeitschriften

Tel.: +49 6221-345-4303

E-Mail: leserservice@springer.com

Suchen Sie noch mehr zum Thema? Mit e.Med - dem Kombi-Abo von Springer Medizin - können Sie schnell und komfortabel in über 600 medizinischen Fachzeitschriften recherchieren und auf alle Inhalte im Volltext zugreifen.

Weitere Infos unter springermedizin.de/eMed 